\title{
A new perspective on optimal care for patients with COPD
}

\author{
*Dirkje Postmaa , Antonio Anzuetob, Peter Calverleyc, Christine Jenkins ${ }^{d}$, Barry J Make , \\ Frank C Sciurba', Thomas Similowski ${ }^{9}$, Thys van der Molen ${ }^{\mathrm{h}}$, Göran Eriksson'
}

a Department of Pulmonary Medicine and Tuberculosis, University Medical Center Groningen, University of Groningen, The Netherlands

${ }^{\mathrm{b}}$ Department of Pulmonology, University of Texas Health Sciences Center, San Antonio, Texas, USA

c Pulmonary and Rehabilitation Research Group, University Hospital Aintree, Liverpool, UK

${ }^{d}$ University of Sydney and Woolcock Institute of Medical Research, Camperdown, Australia

e Division of Pulmonary Sciences and Critical Care Medicine, National Jewish Health, University of Colorado, Denver, USA

${ }^{f}$ Emphysema/COPD Research Center, University of Pittsburgh, Pennsylvania, USA

g Service de Pneumologie et Réanimation, Groupe Hospitalier Pitié-Salpêtrière, Paris, France

${ }^{\mathrm{h}}$ Department of General Practice, University Medical Center Groningen, University of Groningen, The Netherlands

AstraZeneca R\&D, Lund, Sweden

Received 4th February 2011; revised 29th March 2011; accepted 22nd April 2011; online 11th May 2011

\begin{abstract}
Worldwide, clinicians face the task of providing millions of patients with the best possible treatment and management of COPD. Currently, management primarily involves short-term 'here-and-now' goals, targeting immediate patient benefit. However, although there is considerable knowledge available to assist clinicians in minimising the current impact of COPD on patients, relatively little is known about which dominant factors predict future risks. These predictors may vary for different outcomes, such as exacerbations, mortality, co-morbidities, and the long-term consequences of COPD. We propose a new paradigm to achieve 'optimal COPD care' based on the concept that here-and-now goals should be integrated with goals to improve long-term outcomes and reduce future risks. Whilst knowledge on risk factors for poorer outcomes in COPD is growing and some data exist on positive effects of pharmacological interventions, information on defining the benefits of all commonly used interventions for reducing the risk of various future disease outcomes is still scarce. Greater insight is needed into the relationships between the two pillars of optimal COPD care: 'best current control' and 'future risk reduction'. This broader approach to disease management should result in improved care for every COPD patient now and into the future.

(C) 2011 Primary Care Respiratory Society UK. All rights reserved.

D Postma et al. Prim Care Respir J 2011; 20(2): 205-209

doi:10.4104/pcrj.2011.00041
\end{abstract}

Keywords COPD, care, future, management, risk reduction, today

\section{Introduction}

Clinicians are faced with the challenge of providing the best possible management and therapies for patients with COPD. There are several barriers to overcoming this challenge, including the different effects of interventions on individual patients due to heterogeneity within the COPD population, poor relationships between symptoms and indices of disease severity, and limited data on the effects of current management on long-term outcomes in COPD. ${ }^{1,2}$

The pathophysiology of COPD is potentially more complex than that of many other chronic disorders because of considerable disease heterogeneity ${ }^{1}$ and weak relationships between objective measurements used in daily practice (such as the forced expiratory volume in one second $\left[\mathrm{FEV}_{1}\right]$ ) and patient-centred outcomes (such as quality of life and walking distance). ${ }^{2}$ Consequently, more specific information about patient characteristics and more robust, directly informative clinical measurements may be needed to guide COPD management.

Symptoms are notoriously poor predictors of disease diagnosis and severity. ${ }^{2}$ Nevertheless, COPD management in primary care focuses on immediate symptom reduction, mainly resulting from the perceived ineffectiveness of pharmacological treatments to change the progressive course of COPD and the opinion that smoking cessation is the only disease modifier. ${ }^{3,4}$ Although new

\footnotetext{
* Corresponding author: Professor Dirkje Postma, Department of Pulmonary Medicine and Tuberculosis, University Medical Center Groningen, Hanzeplein 1, Groningen, 9713 GZ, The Netherlands. Tel: +31-50-361 3532 E-mail: d.s.postma@long.umcg.nl
} 
data support the efficacy of existing pharmacological treatments on prevention of exacerbations, ${ }^{5,6}$ together with some effect on lung function decline ${ }^{7,8}$ and improved survival, ${ }^{9}$ many clinicians remain sceptical. Additionally, non-pharmacological treatment options in secondary care - including pulmonary rehabilitation, lung volume reduction surgery, oxygen therapy and influenza vaccination - have successfully improved well-being and/or the prognosis of COPD, but are often not considered.

Since current COPD management in general practice still focuses primarily on immediate patient benefit, greater attention to long-term goals should also be advocated as evidence evolves that interventions may impact on long-term disease progression and/or hospitalisations. This is sometimes even independent of any effect they may or may not have on current symptoms. Clinicians already apply this broader approach to risk factors such as hypertension and hypercholesterolaemia in the context of preventing the long-term sequelae of cardiovascular disease. ${ }^{10}$ Treatments to reduce high blood pressure, body mass index, and serum cholesterol are nowadays prescribed independently of any acute effects on current symptoms.

We believe that our knowledge base has progressed to a level where this approach should now also be considered in COPD. Therefore, we propose a new paradigm for the management of COPD based on the concept that 'here-and-now' goals to minimise current impact on an individual patient should be integrated with goals to achieve better long-term outcomes and reduce future risks. The terminologies introduced in this concept paper - 'optimal COPD care', 'best current control', and 'future risk reduction' - have been chosen to reflect a new paradigm of holistic COPD management: the concept that prevention of future risk is of equal and complementary importance to a COPD patient as the immediate impact of treating symptoms. These terms result from extensive discussions between the international group of authors of this article, who have evaluated and considered many terms and terminologies in order to select the best way to present their joint concept.

\section{Optimal COPD care}

Currently, guidelines address the importance of treatment to reduce symptoms and prevent exacerbations (see Figure 1). The latest issue of the Global Initiative for Chronic Obstructive Lung Disease (GOLD) guidelines ${ }^{4}$ states, "Management of COPD continues to be presented in four components: 1) Assess and Monitor Disease, 2) Reduce Risk Factors, 3) Manage Stable COPD and 4) Manage Exacerbations." These terms reflect current disease state, which may have beneficial effects in the future. However, there is no clear distinction between current and future management goals with respect to today's implementation and future risk reduction. There is considerable knowledge of how to minimise the current impact of COPD on a patient's activities of daily living and quality of life, but we need more insight about which ongoing factors determine future outcomes and which treatments may help to minimise the future negative impacts of these factors.

To implement a management strategy aiming at improving future outcomes in COPD it is necessary to define the following:

1) Which outcomes are targeted - for example, reduction of exacerbation risk and medication side effects, prevention of future co-morbidities, COPD complications, loss of health status, lung function and structure, and/or mortality.

2) Which factors determine each future outcome.

3) Which combination of these risk factors has greater predictive ability than any individual measure.

4) Which effective interventions influence these factors with an ultimately meaningful impact on long-term clinical outcomes.

Many contributing factors - such as genetics/ pharmacogenomics, epigenomics, and downstream molecular and cellular mechanisms - are currently unknown or not quantified, and may provide opportunities to enhance the value of algorithms defining the short-term and long-term natural history or potential disease-modifying or adverse effects of specific therapeutics. Addressing both aspects in 'optimal COPD care' will enhance our understanding of these relationships (see Figure 2).

Figure 1. Present guidelines have elements of good COPD control.

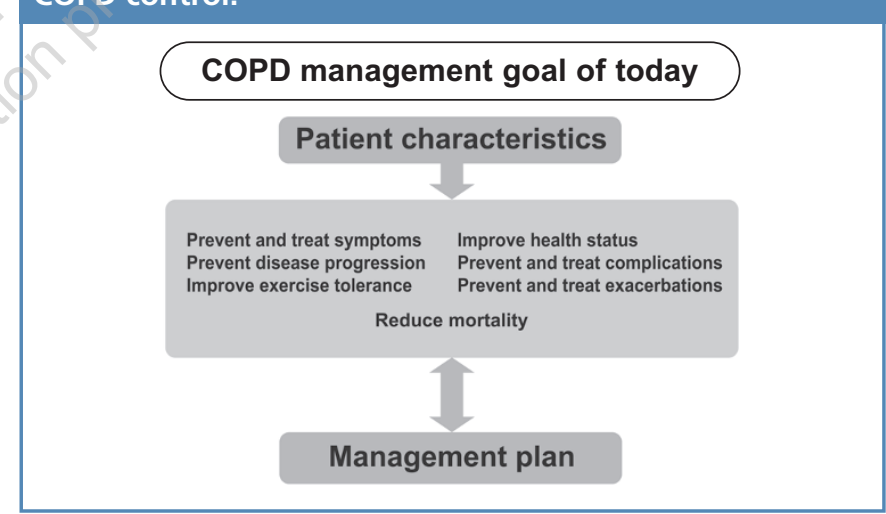

Figure 2. Achieving optimal COPD care encompasses present and future management goals.

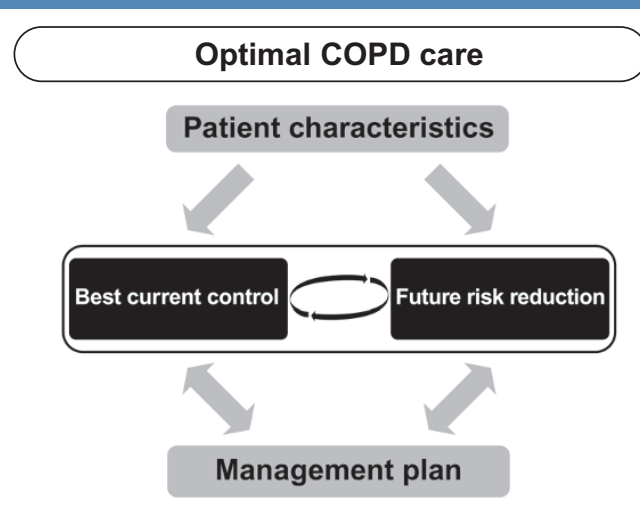




\section{Best current control}

Following initial clinical assessment for potential COPD it is important to identify patient characteristics that are important determinants of disease impact and other factors that contribute to disease severity (see Figure 3). There may be one or more reasons for a patient to seek healthcare, including COPD symptoms, respiratory tract infection, smoking-related symptoms, general health, co-morbidities, or disease-related complications (weight loss, right heart failure, depression, deconditioning). As a next step, demographic, historical and clinical characteristics, and objective measures like lung function and physical activity ${ }^{11}$ should be established (Figure 3). Disease impact on an individual's daily life and well-being, as well as co-morbidities, complications, and medication use, should be evaluated. ${ }^{4}$

Based on these initial assessments, an early general decision can be made to provide optimal management for the presenting symptoms, addressing immediate patient needs (as perceived both by the patient and the clinician) and including a range of strategies of which many have impact and benefits beyond the patient's short-term requirements. Immediate management options may include smoking cessation, pharmacological treatment, rehabilitation, education and psychological support, oxygen therapy, and surgical intervention (as appropriate), in addition to management of co-morbidities and complications, ${ }^{4}$ all with the intention of attaining a patient's best possible current health status. To achieve this, a management approach focusing on dayto-day goals must be tailored to the level of disease severity and impact, and patient needs.
After a patient's initial presentation, clinicians should follow and evaluate the patient's current status, including symptoms, treatment type, reliever use, daily activities, health status, lung function, co-morbidities/complications, and any change in the initially-assessed patient characteristics (Figure 3). Air trapping, ${ }^{12}$ hyperresponsiveness, ${ }^{13}$ and inflammatory or genetic markers, ${ }^{14}$ though currently not appropriate in general practice or not available/validated, may also be assessed during follow-up to elucidate factors contributing to symptoms and optimal therapy. Together, these factors should guide physicians in designing a management plan aimed at achieving best current control.

Elements that constitute a patient's best current control status should be evaluated at every visit in order to guide future management; that is, it is an iterative process.

\section{Future risk reduction}

Managing this pillar of the optimal COPD care concept is challenging. However, careful consideration of each patient's long-term outcomes is critically important, and risk reduction should be an integral part of COPD management. The future impact of COPD encompasses exacerbations, medication side effects, co-morbidities and complications, loss of health status, lung function and lung structure, and mortality (Figure 3). All these future outcomes are risk-related; that is, there is a certain probability of their occurrence. These risks can be categorical (exacerbation risk and mortality) or measurable only over long periods of time (rate of health status/lung function decline). Management of future risk reduction should then be based on

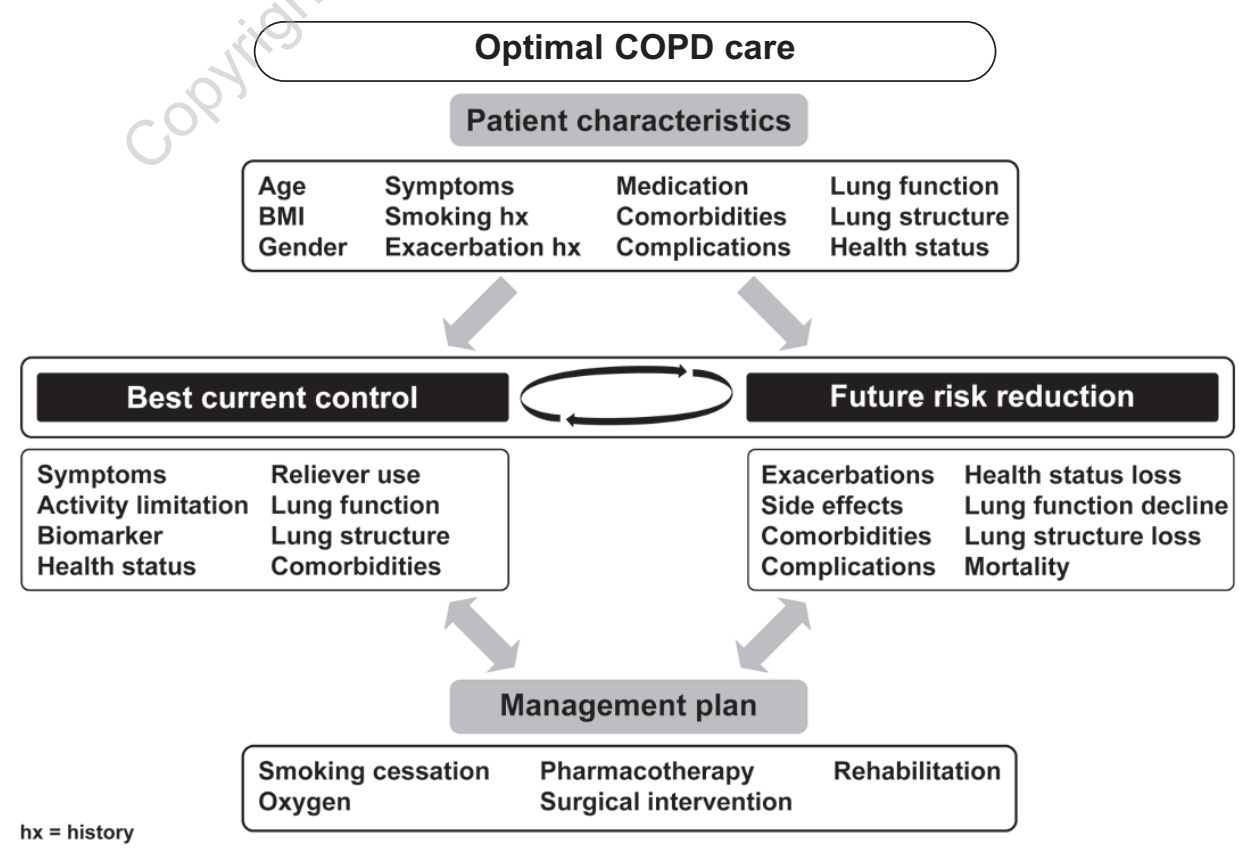


information gathered from patient characteristics and best current control, combined with treatment options that successfully reduce future risks - for example, smoking cessation for lung function decline, ${ }^{3}$ pharmacological treatment for exacerbations, ${ }^{4}$ and pulmonary rehabilitation for hospital admissions and physical condition. Elucidation of predictive (bio)markers is urgently needed in order to facilitate the development of real disease-modifying medication(s) addressing components of the future risk reduction domains.

\section{Aiming for both best current control and future risk reduction to achieve optimal COPD care}

A key initial assumption for this concept is that optimising best current control at all times is beneficial, bearing in mind that the magnitude of benefit achieved is dependent on COPD severity and heterogeneity, the target outcome measurement, and on the adverse effects of medications that need to be minimised.

Another key assumption is that best current control influences future risk and, if achieved, significantly reduces the unnecessary physician contacts, emergency room visits or hospitalisations, and the deterioration in physiological and health status that characterise COPD. Consequently, the opposite assumption should also hold true: poor control today predicts increased risk for disease progression, morbidity, and mortality. Although logical and intuitively appealing, both these hypotheses need to be more thoroughly researched. Today, evidence supporting the concept of the optimal COPD care paradigm is insufficient to be incorporated into clinical practice in all aspects, particularly since we have limited insights into this approach at an individual patient level.

\section{Exacerbations as an example of the link between best current control and future risk reduction}

Acute exacerbations represent a major cause of health status decline and healthcare utilisation in COPD. ${ }^{5,6,15-18}$ Recent studies suggest that exacerbation risk is related to previous exacerbation history, physical activity levels, presence of particular comorbidities such as gastro-oesophageal reflux, and disease severity. ${ }^{19}$ Current therapies are known to decrease the frequency of these future exacerbation events. ${ }^{17,18}$ Therefore, treatment of exacerbations exemplifies the first part of our proposed paradigm; that is, current treatment is warranted independent of its impact on 'best current control' and rather as a target for 'future risk reduction'. This is also exemplified by the insight that the combination of long-acting bronchodilators with inhaled corticosteroids reduces exacerbations and that those treated with placebo have a larger dropout rate due to lack of effective treatment. We need studies in general practice to assess which phenotype best predicts this benefit on an individual level and which treatment type is the best at preventing new exacerbations. This signifies the importance of the proposed paradigm and should be applied to other disease characteristics or co-morbidities that may not be associated with current symptoms.

Is the available data on exacerbations sufficient to bring the 'optimal COPD care' concept to clinical practice? Not fully, since treatment may reduce the number of exacerbations but not necessarily affect lung function decline in the same patient. In addition, even if this is the case in a subset of COPD patients, ${ }^{7.8}$ it is not clear which characteristics predict this response. This also holds true for mortality as a future risk: here we have information that a composite measure such as the BODE index predicts mortality better than airflow obstruction alone and at the same time predicts future exacerbations. ${ }^{20}$ Whether other composite scores are superior to BODE, and whether this differs with other future outcomes, is still unknown.

Thus, depending on the outcome under study, we need greater insight into ways to improve COPD management for now and in the future. This may require studies in large groups of patients characterised for many different phenotypes and followed for some years, applying a Bayesian approach to gather information in order to elucidate the best combination of clinical, physiological, morphological, molecular, or cellular characteristics that reflect the best prognostic risk or therapeutic response indicator for each outcome domain. Also, additional information is required to identify which treatments can potentially reduce certain risk factors.

\section{Clinical research needed}

We need more insight in several clinical categories that impact on current and future outcomes of COPD in order to fill the knowledge gaps regarding the interplay between the two domains of optimal COPD care, 'best current control' and 'future risk reduction'. Such categories include family history, symptoms like sputum expectoration, variability in lung function and clinical expression over days/weeks, health status, lung status (including lung function and structural changes), co-morbidities and complications, smoking cessation, and inflammatory/genomic markers. Questions to be answered for each category are:

- what is the role of each current clinical target and its predictive contribution to 'best current control' and 'future risk reduction' (preferably both)?

- how can these factors be tackled by each specific intervention and what is the effect size?

- how much heterogeneity is there in these responses, and is it predictable in individuals with particular clinical profiles?

Designing research studies to interrogate and inform clinicians in order to fill these knowledge gaps will refine our understanding of disease predictors and their modification in the 
context of optimal COPD management.

Accepting and including this concept of current and future management of COPD is a practical challenge for clinicians, researchers, health managers, government regulators and pharmaceutical companies. Implementation of this concept, combined with careful recording and analysis of outcomes, may lead to a better understanding of COPD heterogeneity and in turn lead to optimised disease management and reduction in future adverse outcomes. Research on relationships between current control and future risk in COPD is needed urgently to inform such a strategy.

\section{Author contributions}

All authors have discussed the development of the new paradigm for COPD management at length and reviewed the literature available. All authors contributed to writing and revising the manuscript.

\section{Funding \& Acknowledgement}

The meetings leading to this paper were funded by AstraZeneca. Editorial assistance (copy proofing, styling, referencing, checking artwork) was provided by Manda Gent, MediTech Media, Rice Street, Manchester, M3 4JL, UK (manda.gent@meditechmedia.com), which was also funded by AstraZeneca.

\section{Conflict of interest declarations}

All authors have completed the Unified Competing Interest form at www.icmje.org/coi_disclosure.pdf (available on request from the corresponding author) and declare:

DSP, PMC, CJ, BJM and TS received financial support for travel to discussions about the submitted work from AstraZeneca (AZ).

DSP has received grants from AZ, GlaxoSmithKline (GSK) and Nycomed.

AA has received research grants and/or honoraria from AZ, Bayer-Schering Pharma, Boehringer-Ingelheim (B-I), Dey Pharmaceuticals, GSK, Lilly Pharmaceuticals, Pfizer, Pneuma Pharmaceuticals and Schering-Plough and has acted as a consultant or advisory board member for AZ, Bayer-Schering Pharma, B-I, Dey Pharmaceuticals, Forest Laboratories, GSK, and Sepracor and has been paid for developing educational presentations for B-I, Dey Pharmaceuticals and GSK.

PMC has been paid for consultancy for $A Z$, has received honoraria for lectures from $A Z$, GSK and Nycomed, provided expert testimony for Forest Laboratories and Nycomed and has been an advisory board member for B-I, GSK, Novartis and Nycomed.

$\mathrm{CJ}$ has contributed to advisory boards and been paid for lectures and development of educational materials for AZ, B-I, GSK, Novartis, Nycomed, Pfizer and Tyrian.

BJM has contributed to advisory boards for AZ, Dey Pharmaceuticals, Embryon, Forest Laboratories, Johnson and Johnson, Novartis, Nycomed, Respironics, Schering and Sequal, has been paid for consultancy for Astellas, Chiesi and Talecris and has received grants or honoraria for lectures from AZ, B-I, GSK, NABI and Pfizer.

FCS has contributed to advisory boards for AZ, GSK, Merck and PnuemRx and has been paid for consultancy by Pfizer and received grants from B-I, GSK and Pfizer.

TS has contributed to advisory boards for AZ, B-I and Nycomed and received a grant from Novartis and has been paid for lectures or educational presentations by AZ, B-I and Novartis.

TvdM has no support or financial relationships with any organisations that might have an interest in the submitted work.

GE is an employee of AZ and holds shares in the company, and is associated with the University Hospital in Lund, Sweden.

\section{References}

1. Rennard SI, Vestbo J. The many "small COPDs": COPD should be an orphan disease. Chest 2008;134(3):623-27. http://dx.doi.org/10.1378/chest.07-3059

2. Price $D$, Crockett $A$, Arne $M$, et al. Spirometry in primary care case-identification, diagnosis and management of COPD. Prim Care Respir J 2009;18(3):216-23. http://dx.doi.org/10.4104/pcri.2009.00055
3. Anthonisen NR, Skeans MA, Wise RA, Manfreda J, Kanner RE, Connett JE. The effects of a smoking cessation intervention on 14.5-year mortality: a randomized clinical trial. Ann Intern Med 2005;142(4):233-39.

4. Global Initiative for Chronic Obstructive Lung Disease. Global strategy for the diagnosis, management, and prevention of chronic obstructive pulmonary disease. Updated 2009. 2009. Available at: http://goldcopd.org/ Guidelineitem. asp?|1=2\&|2=1\&intld=2003 (accessed 1.3.2010).

5. Wedzicha JA, Calverley PM, Seemungal TA, Hagan G, Ansari Z, Stockley RA. The prevention of COPD exacerbations by salmeterolffluticasone propionate or tiotropium bromide. Am J Respir Crit Care Med 2008;177(1):19-26. http://dx.doi.org/ 10.1164/rccm.200707-9730C

6. Puhan MA, Bachmann LM, Kleijnen J, Ter Riet G, Kessels AG. Inhaled drugs to reduce exacerbations in patients with chronic obstructive pulmonary disease: a network meta-analysis. BMC Med 2009;7:2. http://dx.doi.org/10.1186/17417015-7-2

7. Celli BR, Thomas NE, Anderson JA, et al. Effect of pharmacotherapy on rate of decline of lung function in chronic obstructive pulmonary disease: results from the TORCH study. Am J Respir Crit Care Med 2008;178(4):332-38. http://dx.doi.org/10.1164/rccm.200712-18690C

8. Lapperre TS, Snoeck-Stroband JB, Gosman MM, et al. Effect of fluticasone with and without salmeterol on pulmonary outcomes in chronic obstructive pulmonary disease: a randomized trial. Ann Intern Med 2009;151(8):517-27.

9. Calverley PMA, Anderson JA, Celli B, et al. Salmeterol and fluticasone propionate and survival in chronic obstructive pulmonary disease. N Engl J Med 2007;356(8):775-89. http://dx.doi.org/10.1056/NEJMoa063070

10. Weiner SD, Rabbani LE. Secondary prevention strategies for coronary heart disease. J Thromb Thrombolysis 2010;29(1):8-24. http://dx.doi.org/10.1007/s11239-0090381-8

11. Kocks JWH, Asijee GM, Tsiligianni IG, Kerstjens HAM, van der Molen T. Functional status measurement in COPD: a review of available methods and their feasibility in primary care. Prim Care Respir J 2011 (in press) http://dx.doi.org/10.4104/pcrj.2011.00031

12. Casanova C, Cote C, de Torres JP, et al. Inspiratory-to-total lung capacity ratio predicts mortality in patients with chronic obstructive pulmonary disease. Am J Respir Crit Care Med 2005;171(6):591-97. http://dx.doi.org/10.1164/ rccm.200407-8670C

13. Postma DS, De Vries K, Koeter GH, Sluiter HJ. Independent influence of reversibility of air-flow obstruction and nonspecific hyperreactivity on the long-term course of lung function in chronic air-flow obstruction. Am Rev Respir Dis 1986;134(2):276-80.

14. de Torres JP, Pinto-Plata V, Casanova C, et al. C-reactive protein levels and survival in patients with moderate to very severe COPD. Chest 2008;133(6):1336-43. http://dx.doi.org/10.1378/chest.07-2433

15. Spencer S, Calverley PM, Sherwood BP, Jones PW. Health status deterioration in patients with chronic obstructive pulmonary disease. Am J Respir Crit Care Med 2001;163(1):122-28.

16. Niewoehner $D E$. The impact of severe exacerbations on quality of life and the clinical course of chronic obstructive pulmonary disease. Am J Med 2006;119(10 Suppl 1):38-45. http://dx.doi.org/10.1016/j.amjmed.2006.08.006

17. Wedzicha JA, Seemungal TA. COPD exacerbations: defining their cause and prevention. Lancet 2007;370(9589):786-96.

18. Anzueto A, Sethi S, Martinez FJ. Exacerbations of chronic obstructive pulmonary disease. Proc Am Thorac Soc 2007;4(7):554-64. http://dx.doi.org/ 10.1513/pats.200701-003FM

19. Hurst JR, Vestbo J, Anzueto $A$, et al. Susceptibility to exacerbation in chronic obstructive pulmonary disease. N Engl J Med 2010;363(12):1128-38. http://dx.doi.org/10.1056/NEJMoa0909883

20. Marin JM, Carrizo SJ, Casanova C, et al. Prediction of risk of COPD exacerbations by the BODE index. Respir Med 2009;103(3):373-78. http://dx.doi.org/10.1016/j.rmed.2008.10.004 